\title{
ALMOST NORMAL OPERATORS, THEIR SPECTRA AND INVARIANT SUBSPACES ${ }^{1}$
}

\author{
BY C. R. PUTNAM
}

1. Introduction. This lecture will deal with a few classes of operators which in some sense are close to being normal. For the sake of definiteness the underlying Hilbert space $H$ will be taken to be infinite dimensional and separable. Occasionally, it may be convenient to let $H$ be finite dimensional, but, in this case, the operators considered will usually reduce to normal ones. By an operator $T$ is meant a bounded operator, so that $T$ is linear, is defined on the whole space $H$, and satisfies $\|T x\| \leqq$ const $\|x\|$ for all $x$ in $H$. By the spectrum, $\operatorname{sp}(T)$, of $T$ is meant the set of complex numbers $z$ for which $(T-z I)^{-1}$ fails to exist as a bounded operator. Recall that $T$ is said to be normal if $T^{*} T=T T^{*}$.

It seems not out of place here to remark that the "almost" of the title is a bona fide adverb and not part of some compound adjective describing a new kind of operator. In line with this remark it is noteworthy that many classes of almost normal operators which have received attention can be produced by supplying an appropriate prefix or adverb to the root adjective "normal." A few of these prefixes are quasi, sub, hypo, semi, $\left(G_{1}\right)$ (which appears to be a ringer in this group), para (cf. [11], [19]), some adverbs: "nearly," "vaguely" and others. Suffixes apparently have not caught on as well, although "oid" is popular with various root adjectives preceding it, witness normaloid, convexoid and spectraloid; cf. Halmos [16, p. 114]. For classifications of some almost normal operators, see, e.g., Furuta [11, p. 595], Gustafson [13, p. 37], Stampfli [36, p. 473].

The classes of operators we shall consider here can be arranged as follows, each inclusion being proper:

normal $\subset$ quasinormal $\subset$ subnormal $\subset$ hyponormal

$$
\subset \text { seminormal } \subset\left(G_{1}\right) \text {. }
$$

These will be discussed briefly, with particular consideration to necessary and/or sufficient conditions on the spectrum assuring that the operator is (or is not) normal or has (or does not have) a normal part. Finally, in

\footnotetext{
${ }^{1}$ This paper is an expanded version of an invited address given to the American Mathematical Society in Cleveland, Ohio on November 25, 1972. The work was supported by a National Science Foundation grant; received by the editors December 8, 1972.

AMS (MOS) subject classifications (1970). Primary 47A10, 47A15, 47B15, 47B20; Secondary 47B05, 47B47.

Key words and phrases. Spectra, normal operators, hyponormal operators, subnormal operators, seminormal operators, quasinormal operators, $\left(G_{1}\right)$ operators, invariant subspaces, analytic capacity, continuous analytic capacity.
} 
$\S 7$, we shall consider another class of operators resembling normal ones and which, for want of a better name, will be called of zero lower bound type.

In all classes occurring in the inclusions mentioned above, various thinness properties of the spectrum (e.g., nowhere dense, of plane measure zero, being a subset of a smooth curve) will imply normality. The "invariant subspaces" of the title will for the most part refer to those assured by virtue of normality.

An operator $T$ will be called completely quasinormal (completely subnormal, etc.) if $T$ is quasinormal (subnormal, etc.) and if, in addition, there is no nontrivial reducing space on which it is normal; thus, $T$ fails to have a representation $T=T_{1} \oplus N$ where $N$ is normal.

2. Normal operators. It is well known that normal operators $T$ have spectral resolutions, that is, representations of the form $T=\int z d K_{z}$, where the integral is taken over the complex plane or, if one wishes, over the spectrum of $T$. Among the good things supplied by the spectral resolution are plenty of invariant subspaces of $T$. Recall that a subspace $M$ of $H$ is said to be an invariant subspace of $T$ if $T(M) \subset M$ and nontrivial if $M \neq 0, H$.

If $T$ is any operator, normal or not, it is well known that $\operatorname{sp}(T)$ is a nonempty, compact set of the complex plane. This result goes back to Wintner [45]; in this connection, see Hartman [17, p. 485]. Conversely, if one starts with any compact (nonempty) set $X$, there is a normal operator $T$ for which $\operatorname{sp}(T)=X$. In fact, if $H=l^{2}$, the unilateral sequence space, one need only choose $T=\operatorname{diag}\left(t_{1}, t_{2}, \ldots\right)$ where the numbers $t_{i}$ belong to, and are dense in, the set $X$.

3. Quasinormal operators. An operator $T$ belongs to this class if

$$
T\left(T^{*} T\right)=\left(T^{*} T\right) T .
$$

These operators, under a different name, were first investigated by Brown [3]; see also Halmos [16, Problem 108]. It is clear that a normal operator is also quasinormal. That the converse need not hold can be seen by choosing $T$ to be the unilateral shift, that is, if $H=l^{2}$, the matrix

$$
T=\left(\begin{array}{cccc}
0 & 0 & 0 & \ldots \\
1 & 0 & 0 & \ldots \\
0 & 1 & 0 & \ldots \\
\cdots & \ldots & \ldots & \ldots
\end{array}\right) .
$$

It is easily verified that $T^{*} T=I$, so that (3.1) obviously holds, but

$$
T^{*} T-T T^{*}=\operatorname{diag}(1,0,0, \ldots),
$$


so that $T$ is not normal. It is well-known, and easily verified, that $\operatorname{sp}(T)$ is the closed unit disk.

If $T$ is quasinormal, so are all powers $T^{n}, n=0,1,2, \ldots$ On the other hand, the property does not extend to polynomials in $T$, even to translations $T+t I$. Thus, for instance, if $T$ is the shift of (3.2), then $T+t I$ fails to be quasinormal except when $t=0$.

If $T$ is quasinormal but not normal then $\operatorname{sp}(T)$ has an interior. This fact was noted by Stampfli [36, p. 473]. In fact, Brown [3] has shown that every quasinormal operator has a direct sum representation $T=T_{1} \oplus N$ where $N$ is normal and $T_{1}$ is a dilated shift operator associated with a certain positive operator. Professor Brown has recently pointed out to the author that $T_{1}$ is actually a tensor product and that, as a consequence of Brown and Pearcy [4], $\operatorname{sp}\left(T_{1}\right)$ is the closed disk $\left\{z:|z| \leqq\left\|T_{1}\right\|\right\}$. It is clear that if $c>0$ then $c T$, where $T$ is the shift of (3.2), is completely quasinormal with spectrum $\{z:|z| \leqq c\}$. Hence, a compact set $X$ is the spectrum of a completely quasinormal operator if and only if $X=\{z:|z| \leqq c\}, c>0$.

4. Subnormal operators. $T$ is said to be subnormal on the Hilbert space $H$ if there exists a normal operator $N$ on a Hilbert space $K \supset H$ for which $H$ is invariant under $N$ and $T$ is the restriction of $N$ to this invariant subspace. Thus, subnormal operators are the restrictions of normal operators to invariant subspaces.

It turns out that quasinormal operators are subnormal; see Halmos $[16, p .155]$. That the converse is false follows from the following observations. The shift $T$ of (3.2) is subnormal; in fact, the bilateral shift on the bilateral sequence space defined by

$$
x=\left(\ldots, x_{-1}, x_{0}, x_{1}, \ldots\right) \rightarrow y=\left(\ldots, x_{-2}, x_{-1}, x_{0}, \ldots\right)
$$

is a normal extension. Clearly, the operators $T+t I$, where $T$ is defined by (3.2), are subnormal, but, as noted above, not quasinormal unless $t=0$.

It is easy to see that if $T$ is subnormal with the normal extension $N$, then the powers $T^{n}(n=0,1,2, \ldots)$ are subnormal with normal extensions $N^{n}$. Similarly, polynomials in $T$ are also subnormal, as is also $T^{-1}$ in case $T$ is nonsingular.

If $T$ is subnormal there exists such a thing as the minimal normal extension $N$ of $T$ and, moreover, $\operatorname{sp}(N)$ is a subset of $\operatorname{sp}(T)$; see Halmos [14], [15], [16]. In fact, it turns out that $\operatorname{sp}(T)$ can be obtained by filling in some of the holes (bounded components of the complement) of $\operatorname{sp}(N)$. This was first shown by Bram [2]; for a proof due to S. K. Parrott, see $[16$, p. 310]. It may be noted that the bilateral shift of (4.1) is the minimal normal (unitary) extension of the unilateral shift of (3.2) and that the 
former has spectrum $|z|=1$ while the latter has spectrum $|z| \leqq 1$.

Next, we consider the question as to what compact sets $X$ can be the spectra of subnormal, but not normal, operators. To this end, let $X$ denote any compact set and let $C(X)$ and $R(X)$ denote respectively the class of continuous functions on $X$ and the class of functions on $X$ which can be uniformly approximated by rational functions with poles off $X$. In this connection, see, for instance, Gamelin [12] or Zalcman $[\mathbf{4 6}]$.

It was first shown by Hartogs and Rosenthal $[18]$ that $C(X)=R(X)$ in case $X$ has planar measure 0 . This fact can be used, together with an argument given in Wermer [44], to show that if $T$ is subnormal and if $\operatorname{sp}(T)$ has measure 0 then necessarily $T$ is normal. Another proof of this result can be given using the concept of spectral set. Recall that a spectral set (von Neumann [23], see also Halmos [16, p. 123]) of an operator $T$ is a compact set $X$ containing $\operatorname{sp}(T)$, for which $\|f(T)\| \leqq \sup \{|f(z)|: z$ in $X\}$, where $f(z)$ is any rational function with poles off $X$. If $T$ is subnormal then $\operatorname{sp}(T)$ is a spectral set of $T$. Also, if $\operatorname{sp}(T)$ is a spectral set of $T$ and if $C(\operatorname{sp}(T))=R(\operatorname{sp}(T))$ then $T$ is normal; see von Neumann [23], also Lebow [21, p. 73]. Thus if $T$ is subnormal and if its spectrum has measure 0 then $T$ must be normal. This fact apparently was first noted in the literature by Stampfli $[36$, p. 473]. That the result was contained implicitly in Wermer [44] and that it could also be obtained via spectral sets, as indicated above, was pointed out to the author by J. G. Stampfli several years ago.

Recently, it was shown in Clancey and Putnam [7] that, more generally, a compact set $X$ is the spectrum of a subnormal, but not normal, operator if and only if $C(X) \neq R(X)$. Moreover, even a local version of the result is true. Thus, a compact set $X$ is the spectrum of a completely subnormal operator if and only if

$$
C\left(X \cap D^{-}\right) \neq R\left(X \cap D^{-}\right) \quad\left(D^{-}=\text {closure of } D\right),
$$

where $D$ is any open disk intersecting $X$ in a nonempty set.

It may be observed that a necessary condition that $C(X)=R(X)$ is that $X$ be nowhere dense. This is clear from the fact that on an open set only analytic, rather than just continuous, functions can be uniformly approximated by analytic functions, and hence $X$ cannot have any interior. Further, $C(X)=R(X)$ holds for "most" nowhere dense compact sets $X$, in particular, if $X$ has measure 0 (Hartogs and Rosenthal [18]) or if, for instance, $X$ has a finite number of holes. However, there do exist nowhere dense compact sets $X$ (e.g., Mergelyan Swiss cheeses) satisfying (4.2). Such a set can be obtained by removing from the closed unit disk a sequence of open disks, with pairwise disjoint closures, the sum of whose 
radii is finite, in such a way that the remaining (compact) set is nowhere dense. For details, see Gamelin [12, p. 25] or Zalcman [46, p. 69].

Concerning the beautiful results of A. G. Vitushkin and, in particular, associated geometric conditions on a compact set assuring that $A(X)=R(X)$ (where $A(X)$ denotes the set of functions continuous on $X$ and analytic in its interior), that is, if $X$ is nowhere dense, $C(X)=R(X)$, see Zalcman [46, p. $112 \mathrm{ff}]$.

5. Hyponormal and seminormal operators. An operator $T$ is hyponormal if

$$
T^{*} T-T T^{*} \geqq 0
$$

and seminormal if either $T$ or $T^{*}$ is hyponormal. For properties of these operators, cf. Halmos [16], Putnam [26]. If the Hilbert space is finite dimensional then all seminormal operators are normal; this can be seen by noting that the trace of $T^{*} T-T T^{*}$ must be 0 . Further, if $T$ is subnormal then it is also hyponormal. In fact, in the notation of the beginning of $\S 4$, let $P$ denote the orthogonal projection of $K$ onto $H$. If $x, y$ are in $H$ then $\left(T^{*} x, y\right)=(x, T y)=(x, N P y)=\left(P N^{*} x, y\right)$, so that $T^{*} x=P N^{*} x$ for $x$ in $H$. Consequently, for $x$ in $H,\left\|T^{*} x\right\| \leqq\left\|N^{*} x\right\|$ $=\|N x\|=\|T x\|$, that is, (5.1).

That there exist hyponormal operators which are not subnormal was first shown by Halmos [14] (see also [16]), who gave an example of a hyponormal $T$ for which $T^{2}$ was not hyponormal, and hence $T$ was not subnormal. Various other examples are known; for instance, Stampfli [37, p. 378] has given an example of a hyponormal $T$, not subnormal, such that all powers $T^{2}, T^{3}, \ldots$ are subnormal. Incidentally, if $T$ is hyponormal and if $T^{n}$ is normal for some positive integer $n$ then $T$ is normal; see Stampfli [36]. It has been shown by Joshi [20], using results of Stampfli [37], that for every positive integer $n$ there exists a hyponormal, not subnormal, operator $T$ with the property that all polynomials in $T$ of degree not greater than $n$ are hyponormal. The question remains open as to whether the assumption that all polynomials in $T$ are hyponormal implies that $T$ must be subnormal.

As noted in $\$ 4$ above, the spectrum of a subnormal operator is a spectral set of the operator. In addition, if all rational functions of a hyponormal $T$ are also hyponormal, then $\operatorname{sp}(T)$ must be a spectral set of $T$; cf. Clancey and Putnam [8, p. 199]. It may be noted that if $T$ is hyponormal and nonsingular then so is $T^{-1}$; see Stampfli [36, p. 469]. It is unknown, however, whether the hyponormality of all polynomials in $T$ implies that of rational functions of $T$. Further, it may be noted that there exist hyponormal operators $T$ whose spectra are not spectral sets of $T$; see Clancey [6], Wadhwa [43]. Also, examples with totally disconnected 
spectra can be given; see Putnam [30, p. 707], also [33] and the remarks at the end of this section.

As noted above, if $T$ is subnormal, then so are all polynomials in $T$, but the corresponding assertion for hyponormal operators is false. However, there does exist a method for producing a useful variety of hyponormal operators starting from a given one, $T$. Let $T=A+i B$ denote the Cartesian representation of a hyponormal $T$, and let $A=\int u d E_{u}$ denote the spectral resolution of $A=\operatorname{Re}(T)$. If $\beta$ is any Borel set of the real line, it is easy to show (cf. [26, p. 49]) that $E(\beta) T E(\beta)$ is hyponormal on the Hilbert space $E(\beta) H$. It is known (see Putnam [25], also [26, p. 46]) that the spectra of the real and imaginary parts of $T$ are just the projections onto the real and imaginary axes (regarded as real sets) of $\operatorname{sp}(T)$. It follows that the projection of the spectrum of $E(\beta) T E(\beta)$ onto the real axis lies in the closure of $\beta$.

Further, it turns out that the spectrum of $T$ can be specified in terms of the spectra of its real and imaginary parts $A$ and $B$ as follows. Let $\alpha$ denote any open interval containing the real number $t$ and consider the selfadjoint operator $E(\alpha) B E(\alpha)$, which is the imaginary part of $E(\alpha) T E(\alpha)$. Then the spectrum of $T$ lying over $t$, that is, the intersection of $\operatorname{sp}(T)$ with the line $\operatorname{Re}(z)=t$, is precisely the set $\{$ is : $s \in \operatorname{sp}(E(\alpha) B E(\alpha))$ for all $\alpha\}$; see Putnam [32].

If $T$ is hyponormal then

$$
\pi\left\|T^{*} T-T T^{*}\right\| \leqq \operatorname{meas}_{2}(\operatorname{sp}(T)) .
$$

This was proved in Putnam [28]; in case $T^{*} T-T T^{*}$ is compact the result was proved by Clancey [5]. (For an inequality related to (5.2), see Putnam [31].) It follows that if $T$ is hyponormal, but not normal, then its spectrum has positive planar measure. In fact, it was shown in [28] that a local version of this is valid. Thus, if $T$ is completely hyponormal and if $D$ is any open disk intersecting $\operatorname{sp}(T)$, necessarily the set $\operatorname{sp}(T) \cap D$ has positive measure. In fact, if $\operatorname{sp}(T) \cap D$ is a nonempty set of zero planar measure there exists a normal operator $N$ for which $T=T_{1} \oplus N$ and $\operatorname{sp}(N)$ is the closure of $\operatorname{sp}(T) \cap D$.

Recently, it has been shown that, conversely, if $X$ is any compact set with the property that

$$
\text { meas }_{2}(X \cap D)>0 \text { whenever } X \cap D \text { is nonempty } \quad(D=\text { open disk }),
$$

then there exists a completely hyponormal operator $T$ such that $\operatorname{sp}(T)=X$; see Putnam [33]. Thus, a compact set $X$ is the spectrum of a completely hyponormal operator if and only if (5.3) holds.

6. $\left(G_{1}\right)$ operators. If $T$ is any operator it is easily shown that $\left\|(T-z I)^{-1}\right\| \geqq 1 / \operatorname{dist}(z, \operatorname{sp}(T))$ for $z \notin \operatorname{sp}(T)$. An operator $T$ is said to 
satisfy the first order growth condition $\left(G_{1}\right)$ in case equality holds so that

$$
\left\|(T-z I)^{-1}\right\|=1 / \operatorname{dist}(z, \operatorname{sp}(T)), \quad z \notin \operatorname{sp}(T) .
$$

Since the equality (6.1) is one of the basic properties of seminormal operators, the latter are contained in the class $\left(G_{1}\right)$. That the inclusion is proper can be seen from the examples below where $T$ fails to be seminormal.

In case the underlying Hilbert space is finite dimensional, the property $\left(G_{1}\right)$ is known to imply normality. See Stampfli [36], where it is also shown that $T$ may be compact and satisfy (6.1) but fail to be normal. To construct such an example, let $T_{1}$ be defined by

$$
T_{1}=\left(\begin{array}{ll}
0 & 1 \\
0 & 0
\end{array}\right) \quad \text { so that } \quad\left(T_{1}-z I\right)^{-1}=\left(\begin{array}{cc}
-z^{-1} & -z^{-2} \\
0 & -z^{-1}
\end{array}\right) \quad(z \neq 0)
$$

Note that $\operatorname{sp}\left(T_{1}\right)$ on the two-dimensional Hilbert space $\mathrm{H}_{2}$ is the singleton $\{0\}$ and that $T_{1}$ fails to satisfy (6.1). However, by choosing an appropriate normal operator $N$ on an infinite-dimensional space $H_{\infty}$, one can arrange that $T=T_{1} \oplus N$ is compact on $H=H_{2}+H_{\infty}$ and satisfies (6.1).

The question arises as to what compact sets $X$ have the property that if $T$ satisfies (6.1) and if $\operatorname{sp}(T)=X$ then $T$ is normal. In this connection, it was shown by Nieminen [24] that if (6.1) holds and if $\operatorname{sp}(T)$ is real then $T$ is selfadjoint, and by Donoghue [10] that if (6.1) holds and if $\operatorname{sp}(T)$ is a subset of $|z|=1$ then $T$ is unitary. These results were generalized by Stampfli [38], [39], who showed that if $T$ is $\left(G_{1}\right)$, or even locally $\left(G_{1}\right)$, so that (6.1) is assumed to hold only for $z$ belonging to $U-\operatorname{sp}(T)$, where $U$ is some open set containing $\operatorname{sp}(T)$, and if $\operatorname{sp}(T)$ is, for instance, a subset of a piecewise $C^{2}$ curve (having continuous right- and left-hand tangents where the smooth pieces join) then necessarily $T$ is normal.

This furnishes only a sufficient condition for normality. It may be noted however that Stampfli [38, p. 9] has shown that if $T_{1}$ is the matrix of (6.2) then a normal operator $N$ can be chosen so that $T=T_{1} \oplus N$ satisfies (6.1) and $\operatorname{sp}(T)$ lies on a curve $f(t)$, where $f(t)$ is continuous on $0 \leqq t \leqq 1$ and has a continuous second derivative on $0<t \leqq 1$. One need only choose $f(t)$ so as to satisfy $\operatorname{dist}(z, f(t)) \leqq|z|^{-2}$ for $0<z<1$ (cf. (6.2)) and the required smoothness conditions. The question remains open as to a necessary and sufficient condition on a compact set in order that it be the spectrum of a completely $\left(G_{1}\right)$ operator, that is, one having no normal part. For other results relating to the $\left(G_{1}\right)$ condition see also Luecke [22], Putnam [27], Stampfli [40], [41], and the references contained there.

7. Zero lower bound operators. This last section will deal with another 
class of operators resembling normal operators. Let $T_{z}=T-z I$ and suppose that $D$ is any nonnegative selfadjoint operator satisfying

$$
T_{z} T_{z}^{*} \geqq D \geqq 0 \quad \text { for all } z \text {. }
$$

It was shown in Putnam [34] that if $D \neq 0$ and if $z$ is not in the set $P$, where

$$
P=\left\{z: z \text { in the point spectrum of } T \text { or of } T^{*}\right\},
$$

then there exists a vector $x \neq 0$ for which

$$
\left\|(T-z I)^{-1} x\right\| \leqq \text { const }\|x\|,
$$

and, if $y$ is any vector in $H$,

$$
\left((T-z I)^{-1} x, y\right) \text { is continuous and bounded on } C-P \text {. }
$$

It follows from this (cf. [34]), using a result in [29], that $T$ cannot then be normal ; in particular, if $T$ is normal and if (7.1) holds, necessarily $D=0$. We shall call an operator a zero lower bound (ZLB) operator if (7.1) holds only for $D=0$; thus, in particular, every normal operator is of this class.

It is easily verified that $T$ satisfies (7.1) with a $D \neq 0$ if $T^{*}$ is hyponormal but not normal. That these latter operators are properly contained in the set of non-ZLB operators can be seen by choosing $T^{*}$ to be the direct sum of a nonnormal hyponormal operator and an arbitrary operator.

Next, we need the notions of analytic capacity $\gamma(X)$ and continuous analytic capacity $\alpha(X)$ of a compact set $X$. For definitions and properties, see Gamelin [12, Chapter 8], or Zalcman [46, p. $11 \mathrm{ff} ., 77 \mathrm{ff}]$. The idea of continuous analytic capacity (or AC capacity) was introduced by Dolzhenko [9]; see [46, p. 143], and the references mentioned there, in particular, Arens [1]. It turns out that a compact set of analytic capacity 0 must also have continuous capacity 0 , but that, in general, the converse is false. Further, compact sets of analytic capacity 0 are just the removable compact sets for bounded analytic functions. Also, if $X$ is compact and of planar measure 0 then necessarily $\alpha(X)=0$ but the converse need not hold.

Let $P^{-}$denote the closure of the set $P$ of (7.2). It was shown in [34] that $T$ is a ZLB operator if either

$$
\gamma\left(\operatorname{sp}(T) \cup P^{-1}\right)=0
$$

or

$$
\gamma\left(P^{-1}\right)=0 \quad \text { and } \quad \alpha\left(\operatorname{sp}(T) \cup P^{-}\right)=0 .
$$

For instance, compact operators $T$ are of ZLB type since (7.5) (or (7.6)) 
is satisfied. Also, if $T$ is seminormal, so that $T$ or $T^{*}$ is hyponormal, and if either (7.5) or (7.6) holds, then $T$ must be normal. However, as noted in $\S 5$, if $T$ is seminormal, the same conclusion holds even when $\operatorname{sp}(T)$ is any set of planar measure 0 , whether or not (7.5) or (7.6) holds.

As noted above, either (7.5) or (7.6) is sufficient to ensure that $T$ be a ZLB operator. However, it does not seem to be known, for instance, whether every compact set of planar measure 0 but of positive continuous analytic capacity is the spectrum of some non-ZLB operator, or, even better, of some operator having no nontrivial reducing space on which it is a ZLB operator.

Finally, it may be mentioned that if $T$ is a contraction $(\|T\| \leqq 1)$ satisfying (7.1) with $D \neq 0$ and if

$$
\left\{T^{n}\right\}_{n=1,2, \ldots} \text { does not converge strongly to } 0 \text {, }
$$

then, as a consequence of (7.3) and (7.4) above (see [34]), $T$ has a nontrivial invariant subspace. In case $T^{*}$ is subnormal then (7.7) implies that $\left\{T^{* n}\right\}_{n=1,2, \ldots}$ also does not converge to 0 , and the assertion can be deduced from the Sz.-Nagy and Foias theorem (see [42, p. 314], also $[16$, p. 96]). However, the latter theorem does not appear to be applicable when the contraction $T$ is assumed only to satisfy (7.1) with $D \neq 0$, even if $T^{*}$ is hyponormal (again, with $D \neq 0$, as can be supposed if $T^{*}$ is not normal).

\section{REFERENCES}

1. R. Arens, The maximal ideals of certain function algebras, Pacific J. Math. 8 (1958), 641-648. MR 22 \#8315.

2. J. Bram, Subnormal operators, Duke Math. J. 22 (1955), 75-94. MR 16, 835.

3. A. Brown, On a class of operators, Proc. Amer. Math. Soc. 4 (1953), 723-728. MR 15, 538.

4. A. Brown and C. Pearcy, Spectra of tensor products of operators, Proc. Amer. Math. Soc. 17 (1966), 162-166. MR 32 \#6218.

5. K. F. Clancey, Seminormal operators with compact self-commutators, Proc. Amer. Math. Soc. 26 (1970), 447-454. MR 42 \# 885.

6. - Examples of nonnormal seminormal operators whose spectra are not spectral sets, Proc. Amer. Math. Soc. 24 (1970), 797-800.

7. K. F. Clancey and C. R. Putnam, The local spectral behavior of completely subnormal operators, Trans. Amer. Math. Soc. 163 (1972), 239-244.

8. - Normal parts of certain operators, J. Math. Soc. Japan 24 (1972), 198-203.

9. E. P. Dolženko, Approximation on closed regions and zero-sets, Dokl. Akad. Nauk SSR 143 (1962), 771-774 Soviet Math. Dokl. 3 (1962), 472-475. MR 24 \# A2670.

10. W. Donoghue, On a problem of Nieminen, Inst. Hautes Études Sci. Publ. Math. 16 (1963), 31-33. MR 27 \# 2864.

11. T. Furuta, On the class of paranormal operators, Proc. Japan Acad. 43 (1967), $594-598$. MR 36 \# 4354.

12. T. W. Gamelin, Uniform algebras, Prentice-Hall, Englewood Cliffs, N.J., 1969.

13. K. Gustafson, State diagrams for Hilbert space operators, J. Math. Mech. 18(1968/69), 33-45. MR 37 \# 3388.

14. P. R. Halmos, Normal dilations and extensions of operators, Summa Brasil. Math. 2 (1950), 125-134. MR 13, 359.

15. , Spectra and spectral manifolds, Ann. Soc. Polon. Math. 25 (1952), 43-49. MR 14, 1096. 
16. P. R. Halmos, A Hilbert space problem book, Van Nostrand, Princeton, N.J., 1967. MR 34 \# 8178.

17. P. Hartman, Aurel Wintner, J. London Math. Soc. 37 (1962), 483-503. MR 25 \#4983.

18. F. Hartogs and A. Rosenthal, Über Folgen analytischer Funktionen, Math. Ann. 104 (1931), 606-610.

19. V. Istratescu, T. Saitô and T. Yoshino, On a class of operators, Tôhoku Math. J. (2) 18 (1966), 410-413. MR 35 \#756.

20. A. D. Joshi, Hyponormal polynomials of monotone shifts, Thesis, Purdue University, Fort Wayne, Ind., 1971.

21. A. Lebow, On von Neumann's theory of spectral sets, J. Math. Anal. Appl. 7 (1963), 64-90. MR 27 \#6149.

22. G. R. Luecke, Operators satisfying condition $\left(\mathrm{G}_{1}\right)$ locally (to appear).

23. J. von Neumann, Eine Spektraltheorie für allgemeine Operatoren eines unitären Raumes, Math. Nachr. 4 (1951), 258-281. MR 13, 254.

24. T. Nieminen, $A$ condition for the self-adjointness of a linear operator, Ann. Acad. Sci. Fenn. Ser. A. I No. 316 (1962), 5 pp. MR 25 \#2452.

25. C. R. Putnam, On the spectra of semi-normal operators, Trans. Amer. Math. Soc. 119 (1965), 509-523. MR 32 \# 2913.

26. - Commutation properties of Hilbert space operators and related topics, Ergebnisse der Mathematik und ihrer Grenzgebiete, Band 36, Springer-Verlag, New York, 1967. MR 36, \# 707.

27. The spectra of operators having resolvents of first-order growth, Trans. Amer. Math. Soc. 133 (1968), 505-510. MR 37 \#4651.

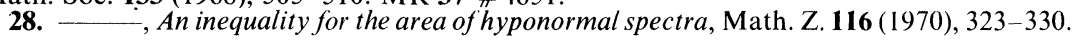
MR 42 \# 5085.

29. Ranges of normal and subnormal operators, Michigan Math J. 18 (1971), 33-36. MR 43 \# 2550.

30. - The spectra of completely hyponormal operators, Amer. J. Math. 93 (1971), 699-708. MR 43 \#6757.

31. Trace norm inequalities for the measure of hyponormal spectra, Indiana Univ. Math. J. 21 (1972), 775-779.

32., A similarity between hyponormal and normal spectra, Illinois J. Math. 16 (1972), 695-702.

33. - - The role of zero sets in the spectra of hyponormal operators (preprint).

34. - Resolvent vectors, invariant subspaces, and sets of zero capacity, Math. Ann. (to appear).

35. J. G. Stampfli, Hyponormal operators, Pacific J. Math. 12 (1962), 1453-1458. MR 26 \#6772.

36. - Hyponormal operators and spectral density, Trans. Amer. Math. Soc. 117 (1965), 469-476. MR 30 \#3375.

37. - Which weighted shifts are subnormal, Pacific J. Math. 17 (1966), 367-379. MR 33 \# 1740.

38. - A local spectral theory for operators, J. Functional Analysis 4 (1969), 1-10. MR 39 \#4698.

39. A local spectral theory for operators. II, Bull. Amer. Math. Soc. 75 (1969), 803-806. MR 39 \#6108.

40. - A local spectral theory for operators. III : Resolvents, spectral sets and similarity, Trans. Amer. Math. Soc. 168 (1972), 133-151.

41. - A local spectral theory for operators. IV: Invariant subspaces, Indiana Univ. Math. J. 22 (1972), 159-167.

42. B. Sz.-Nagy and C. Foiaş, Sur les contractions de l'espace de Hilbert. IX. Factorisations de la function caractéristique. Sous-espaces invariant, Acta Sci. Math. (Szeged) 25 (1964), 283-316. MR 31 \# 5101.

43. B. Wadhwa, A hyponormal operator whose spectrum is not a spectral set, Proc. Amer. Math. Soc. 38 (1973), 83-85.

44. J. Wermer, Report on subnormal operators, Report of an International Conference on Operator Theory and Group Representations, Arden House, Harriman, N.Y., 1955, pp. 1-3, Pub. \# 387, NAS-NRC, Washington, D.C., 1955. MR 17, 880.

45. A. Wintner, Zur Theorie der beschränkten Bilinearformen, Math. Z. 30 (1929), $228-282$.

46. L. Zalcman, Analytic capacity and rational approximation, Lecture Notes in Math., no. 50, Springer-Verlag, Berlin and New York, 1968. MR 37 \# 3018.

Department of Mathematics, Purdue University, West Lafayette, Indiana 47907 